\title{
On Decisions That Lead to Decisions: Direct and Derived Evaluations of Preference
}

\author{
SANJAY SOOD \\ YUVAL ROTTENSTREICH \\ LYLE BRENNER*
}

\begin{abstract}
Many consumer choices lead to subsequent decisions. In such situations, any choice option may be evaluated based on its own characteristics (direct evaluations), or evaluated based on the characteristics of alternatives it makes available in subsequent decisions (derived evaluations). We contrast direct and derived evaluations in the context of two consumer research issues. First, in choices between a lone option and a group of alternatives, direct evaluations bias preferences toward the group, whereas derived evaluations bias preferences away from the group. Second, in choices between stores, sensitivity to price is greater under derived than direct evaluations.
\end{abstract}

$\mathrm{M}$ any consumer decisions arise within a sequence of related decisions (Bettman 1970, 1971, 1979; Bettman and Park 1980; Hauser 1986; Payne 1976). For example, imagine a consumer who receives an unanticipated windfall and considers whether to save this money or spend it on a digital camera. Suppose the consumer decides to purchase the camera. This decision may necessitate another decision concerning whether to purchase the camera online or at a local store. Suppose the consumer decides to make the purchase at a store. This decision, in turn, requires that the consumer decide which store to visit. The decision among stores then leads to the choice of a particular camera from those offered at that store.

In this article, we contrast direct and derived evaluations, two processes by which consumers may assess their options at any particular point within a decision sequence. To illustrate these concepts, consider a version of the scenario just outlined. Suppose that two consumers are deciding whether to visit Circuit City or Best Buy to purchase a digital camera and each consumer finds Circuit City attractive, the first because "it is nearby" and the second because "the topof-the-line Nikon and Minolta camera are on sale there." The first consumer has evaluated Circuit City directly-on the basis of a characteristic of that store (its location). In

*Sanjay Sood is assistant professor of marketing, Anderson Graduate School of Management, University of California, Los Angeles. Yuval Rottenstreich is associate professor of management and organizational behavior, Graduate School of Business, University of Chicago. Lyle Brenner is assistant professor of marketing, Warrington College of Business, University of Florida. The order of authorship is arbitrary. The authors thank the editor, the associate editor, and the three reviewers for their extremely helpful suggestions. contrast, the second consumer has derived an evaluation of Circuit City from considerations of options (the Nikon and Minolta) it makes available in the subsequent choice among cameras.

In general, direct evaluations focus on aspects of current choice alternatives (e.g., the stores Circuit City and Best Buy) that are not properties of any individual subsequent options (e.g., the cameras). For instance, the proximity of a store, the helpfulness of its salespeople, and the easy availability of parking are all aspects of a store, but not of any individual item to be purchased at the store. In contrast, derived evaluations base assessments of current alternatives on properties of the options available in subsequent choices. For instance, one might visit a store because it offers low prices on Nikons and Minoltas, always has the newest Sony models, or sells premium brands.

Note that the same consideration can be either direct or derived depending on where the consumer is within a decision sequence. For example, if the consumer is deciding between stores, choosing Circuit City because the Nikon is on sale there constitutes a derived evaluation of Circuit City. However, once a consumer is at Circuit City and is choosing between cameras, opting for the Nikon because it is on sale constitutes a direct evaluation of the Nikon. Typically, an evaluation that is derived with respect to an initial decision is direct with respect to a subsequent decision.

Because they draw on different considerations, direct and derived evaluations may yield systematically different choices. We explore the implications of this hypothesis for two issues in consumer research. We first investigate situations in which consumers choose between a lone option (e.g., buying the brand recommended by a friend; making dinner at home) and a group of potential alternatives (e.g., 
deciding to buy one of the brands not recommended by one's friend; deciding to go out for dinner at one of the restaurants nearby). Opting for the group necessitates a subsequent decision (e.g., which unrecommended brand to purchase; which restaurant to go to). In several studies of loneversus-group choices, Kahn, Moore, and Glazer (1987; see also Glazer, Kahn, and Moore 1991) observed a systematic bias toward choosing the group, a pattern they termed the lone-alternative effect. However, in another series of studies, Brenner, Rottenstreich, and Sood (1999) observed the opposite pattern, a bias toward choosing the lone option. To make salient the divergent nature of these findings, we refer to the pattern observed by Kahn et al. as group advantage and to the pattern observed by Brenner et al. as group disadvantage. We reconcile these two discrepant results by experimentally demonstrating that one emerges when direct evaluations predominate whereas the other emerges when derived evaluations predominate.

We also investigate the effect of direct and derived evaluations on consumers' price sensitivity. A fundamental premise of the distinction between direct and derived evaluations is that derived evaluations depend on characteristics of the individual alternatives available in subsequent choices, whereas direct evaluations do not. For example, in the camera-purchase scenario, derived evaluations of the stores may depend on the prices of the individual cameras available at each store, but direct evaluations of the stores will not. Thus, when choosing which store to visit, consumers engaging in derived rather than direct evaluations should be more sensitive to changes in the prices of individual cameras.

Our experiments highlight two general determinants of the tendency to engage in direct versus derived evaluations. First, direct evaluations of a choice option are more likely when the option has a meaningful characterization, or a coherent unit identity that the consumer may consider. To illustrate, suppose that a consumer is shown two heterogeneous sets of cameras, set $\mathrm{A}$ and set $\mathrm{B}$, each containing several brands and price levels, and is asked from which set she prefers to make a purchase. Alternatively, suppose that a consumer is told that store A stocks the cameras in set A, is told that store B stocks the cameras in set B, and is asked from which store she prefers to make a purchase. As we have mentioned, the choice pitting two stores may proceed either by direct evaluations (e.g., store A is nearby, store B is open late) or derived evaluations (e.g., store A stocks the new Nikon). In contrast, the choice between the two sets of items is unlikely to rely on direct evaluations. Unlike a store, a heterogeneous set of cameras is not a natural target for direct evaluation. One set is not closer than the other, nor is one set open later than the other, and so forth. The absence of a meaningful characterization diminishes the rationale for direct evaluation, thus encouraging derived evaluation.

Second, independent of the presence or absence of a unit identity for the choice options, the method used to elicit preferences may also influence the tendency to make either direct or derived evaluations. For instance, consider being asked "Would you rather go to store A or store B?" versus "Is the item you prefer at store A or store B?" The first question focuses attention on the stores, whereas the second makes salient the subsequent choice among items. Slovic's (1972) concreteness principle, according to which people typically accept and use information in the form in which it is given to them, suggests that the first question should promote direct evaluations whereas the second question should promote derived evaluations (also see Simon 1983; Slovic, Griffin, and Tversky 1990; Tversky and Kahneman 1986; Tversky, Sattath, and Slovic 1988). In our experiments, we vary both $(a)$ the presence or absence of a meaningful characterization of a group of choice options and $(b)$ the form of the preference query in order to manipulate the degree of direct and derived evaluations.

The remainder of the article is organized as follows. We first review research on group advantage and disadvantage and explain how the distinction between different evaluations can reconcile these opposite findings. Experiment 1 manipulates the tendency toward either direct or derived evaluations to reproduce the dual pattern of group advantage and disadvantage. Next, experiment 2 tests the prediction of greater price sensitivity when consumers engage in derived rather than direct evaluation. We conclude by discussing future directions and by reviewing evidence that suggests that consumers may often rely excessively on direct evaluation.

\section{RESEARCH ON GROUP ADVANTAGE AND DISADVANTAGE}

In studies of lone-versus-group choices, intriguing but opposite biases have been observed by Kahn et al. (1987; see also Glazer et al. 1991), and Brenner et al. (1999). Kahn et al. and Glazer et al. studied decision problems in which participants chose between two stores. In each problem, one store offered a single item (e.g., Coke) while the other store offered a pair of items (e.g., Diet Coke and 7-Up). Participants were asked to imagine that they intended to buy a single item and to indicate at which of the two stores they preferred to make their purchase. In a control condition, no mention was made of any stores and none of the items was singled out from the others; participants were simply asked which of the three items was their favorite. The results revealed group advantage: participants selected the store offering the group of items more often than they selected those items in the control condition.

Brenner et al. examined lone-versus-group choices using a different experimental method. Participants chose among four items without any mention of different stores. They were informed that three of the options had been randomly grouped together and were asked to indicate whether they preferred the lone option or their choice of one of the grouped options. The results revealed group disadvantage: participants showed a bias in favor of selecting the lone option and avoiding the group.

The distinction between direct and derived evaluations 
can be used to reconcile the opposite results of Kahn et al. and Brenner et al. Indeed, Kahn et al.'s analysis of group advantage implicitly invokes direct evaluations, and Brenner et al.'s analysis of group disadvantage implicitly invokes derived evaluations.

Kahn et al. suggest that consumers are biased toward choosing the store offering the group of options primarily because of a preference for flexibility. Perhaps because deciding between the stores is somewhat difficult or because there is no clear favorite item, participants may prefer to leave their options open and opt for what in economics is known as the option value provided by the store offering more items (see Koopmans 1964; Kreps 1979; Peleg and Yaari 1973; Strotz 1955). A preference for flexibility constitutes a direct evaluation; it reflects an assessment of the stores based on an aspect of each store (i.e., offering a small or large selection) that is not a property of any subsequent individual item.

In contrast, Brenner et al.'s explanation of group disadvantage invokes derived evaluations. Brenner et al. argue that consumers making a lone-versus-group choice typically compare each of the grouped items to one another, identify their favorite from the group, and, finally, compare that item to the lone alternative. In such a procedure, the lone alternative endures only one comparison, whereas the group favorite endures several comparisons. Following Tversky and Kahneman (1991) and Tversky and Simonson (1993), Brenner et al. invoke loss aversion to predict that an option's attractiveness will typically be reduced by comparisons to other options. Comparisons between options make salient advantages (gains) and disadvantages (losses) of each option relative to other options (see Hsee and Leclerc 1998; Huber, Payne, and Puto 1982), and loss aversion implies that disadvantages will loom larger than corresponding advantages. Thus, when the items being compared have roughly commensurate advantages and disadvantages, the attractiveness of an option will decrease as it is compared to more options. Because the group favorite is exposed to more comparisons than the lone option, the attractiveness of the group favorite will be reduced more than the attractiveness of the lone option. Consequently, people will tend to avoid the group.

In summary, Kahn et al. studied choices between stores, and explained their result in terms of a preference for flexibility-which implicates direct evaluations. In contrast, Brenner et al. studied choices between groups of items, and explained their result in terms of a particular pattern of interitem comparisons-which implicates derived evaluations. The different tasks employed by these experimenters make salient different considerations: when choosing between stores, store-level characteristics (i.e., direct evaluations) are more salient; when choosing between groups of items, itemlevel characteristics (i.e., derived evaluations) are more salient.

As argued above, direct evaluations of an option are more likely when that option has a meaningful characterization or coherent unit identity. Moreover, labeling an option as a store does provide an overall unit identity for the option, whereas casting an option merely as a set of items does not. Thus, the dual pattern of group advantage and disadvantage may arise because the different tasks used in past research shift the relative balance of direct and derived evaluations. These observations lead to our first hypothesis:

H1: In lone-versus-group choices, direct evaluations will yield group advantage, whereas derived evaluations will yield group disadvantage.

We should note that, in addition to differentially facilitating either direct or derived evaluations, the experiments of Kahn et al. and Brenner et al. differed in numerous other details, for example, by using different stimuli and groups of different sizes. In experiment 1 , we hold such details of the task constant and attempt to manipulate only the tendency to engage in direct or derived evaluations.

\section{EXPERIMENT 1: RECONCILING GROUP ADVANTAGE AND DISADVANTAGE}

\section{Method}

We presented 653 undergraduates at a southeastern university with eight lone-versus-group choice problems, summarized in table 1 . The problems were included in a questionnaire packet along with several unrelated tasks. Each problem involved four options from a particular product category; for example, one problem required choosing between turkey, ham, roast beef, and tuna salad sandwiches for lunch. In each problem, the four options were partitioned into a lone option (L) and a group (G) of three options $\left(\mathrm{g}_{1}\right.$, $\mathrm{g}_{2}$, and $\mathrm{g}_{3}$ ). Participants were asked to imagine that they intended to purchase a single item from each product category. They then indicated preference for either $\mathrm{L}$ or $\mathrm{G}$. Note that the choice between $\mathrm{L}$ and $\mathrm{G}$ occurs in the context of a potential subsequent decision among the three options in $\mathrm{G}$. Consideration of $\mathrm{G}$ may thus proceed by direct evaluation of $\mathrm{G}$ itself or by derived evaluation focusing on $\mathrm{g}_{1}$, $\mathrm{g}_{2}$, and $\mathrm{g}_{3}$ (or by a combination of both types of evaluations).

The experiment included four between-subject conditions. In two of these conditions-the store-direct and store-derived conditions - the options were partitioned into $\mathrm{L}$ and $\mathrm{G}$ on the basis of the store in which they were located. The instructions in these conditions stated that one of the items was available at one store and that the other three items were available at another store. Participants were informed that the two stores were equally far away and offered similar prices. In the two remaining conditions-the item-one-stage and item-two-stage conditions - the options were arbitrarily partitioned into a lone option and a group of three options; no mention was made of any stores. Participants in these conditions were informed that three of the items had been randomly grouped together and separated from the remaining item.

To reiterate, the store conditions include meaningful characterizations of $\mathrm{L}$ and $\mathrm{G}$ as stores, whereas the item conditions cast $\mathrm{L}$ and $\mathrm{G}$ merely as sets of items. Characterization of $\mathrm{G}$ as a store provides a basis and motivation for direct 
TABLE 1

SUM OF LONE OPTION CHOICE SHARES (S, IN \%) FOR EXPERIMENT 1

\begin{tabular}{|c|c|c|c|c|c|}
\hline Category & Options & $\begin{array}{l}\text { Store-direct } \\
(n=217)\end{array}$ & $\begin{array}{l}\text { Store-derived } \\
\quad(n=211)\end{array}$ & $\begin{array}{l}\text { Item-one-stage } \\
\quad(n=101)\end{array}$ & $\begin{array}{l}\text { Item-two-stage } \\
\quad(n=124)\end{array}$ \\
\hline $\begin{array}{l}\text { Sandwiches } \\
\text { Entrees } \\
\text { Cereal } \\
\text { Cookies } \\
\text { Beverages } \\
\text { Candy Bars } \\
\text { Ice Cream } \\
\text { Fruit } \\
\text { Average }^{\text {a }}\end{array}$ & $\begin{array}{l}\text { Turkey, ham, roast beef, tuna salad } \\
\text { Fish, pasta, chicken, beef } \\
\text { Grape Nuts, Cheerios, Frosted } \\
\text { Flakes, Raisin Bran } \\
\text { Fig Newton, Nutter Butter, Oreo, } \\
\text { Chips Ahoy } \\
\text { Gatorade, Fruitopia, Snapple, } \\
\text { Frappuccino } \\
\text { Kit Kat, M\&Ms, Snickers, } \\
\text { Butterfinger } \\
\text { Chocolate, strawberry, vanilla, } \\
\text { cookies and cream } \\
\text { Banana, peach, apple, orange }\end{array}$ & $\begin{array}{l}63 \\
63 \\
\\
73 \\
67 \\
\\
72 \\
51 \\
\\
58 \\
27 \\
59 \\
(3.6)\end{array}$ & $\begin{array}{c}98 \\
83 \\
86 \\
103 \\
105 \\
84 \\
115 \\
56 \\
92 \\
(4.4)\end{array}$ & $\begin{array}{c}123 \\
127 \\
149 \\
92 \\
103 \\
88 \\
\\
127 \\
111 \\
116 \\
(7.6)\end{array}$ & $\begin{array}{c}142 \\
134 \\
116 \\
124 \\
120 \\
88 \\
\\
115 \\
126 \\
122 \\
(6.0)\end{array}$ \\
\hline
\end{tabular}

NOTE.-Values less than 100 indicate group advantage, and values greater than 100 indicate group disadvantage.

astandard errors (in parentheses) are for each average value of $S$ across the eight problems.

evaluation; in the absence of such a meaningful characterization of $\mathrm{G}$, there is less rationale for direct evaluation and derived evaluations should predominate. Furthermore, while many different direct and derived evaluations are possible, in the present context, direct evaluations are predicted to reflect the preference for flexibility identified by Kahn et al., yielding group advantage. In contrast, derived evaluations are predicted to involve the item-by-item comparisons outlined by Brenner et al., yielding group disadvantage.

The two store conditions differed from one another in terms of the question used to elicit preferences. The storedirect condition $(n=217)$ was patterned after the methodology used by Glazer et al. For each problem, the instructions in this condition asked, "Which store would you rather go to?" and participants placed a check mark next to either "I would rather go to Store A (the store with one option)" or "I would rather go to Store B (the store with three options)." The store-derived condition $(n=211)$ altered this procedure by directing more attention to the individual items. Participants were asked to indicate "whether the item you want is at the first or second store," and placed a check mark next to either "I would rather buy [item w] . . . at Store A" or "I would rather buy either [item x, y, or $\mathrm{z}$ ] at Store B." Both conditions provide a meaningful characterization of $\mathrm{L}$ and $\mathrm{G}$ as stores, but because the storederived condition focuses more closely on the individual items, it should yield more derived evaluations and hence less group advantage. In neither condition were participants required to indicate their preferred item from the group.

The two item conditions also differed from one another in terms of the question used to elicit preferences. The itemone-stage $(n=101)$ condition replicated the procedure of Brenner et al. Participants were asked to indicate "whether you prefer the lone option or your choice of one of the other three." In this task Brenner et al. suggested that participants engage almost exclusively in derived evaluations, by com- paring the items in the group, identifying a favorite, and then comparing the group favorite with the lone option. Because of loss aversion, this procedure should reduce the attractiveness of the group favorite and yield group disadvantage. The item-two-stage condition $(n=124)$ was designed to test this analysis by explicitly requiring the pattern of comparisons suggested by Brenner et al. Participants were initially presented with only the three options making up the group and indicated their favorite of the three. Then participants were presented with the fourth option and indicated whether they preferred it or their previously identified group favorite. If Brenner et al.'s analysis is correct, the one- and two-stage conditions should reveal about the same degree of group disadvantage, because the same sequence of comparisons occurs in both cases.

To summarize, the four conditions differ in (i) whether they offer meaningful characterizations of the options that facilitate direct evaluations and (ii) the degree to which the question asked of participants draws attention to individual items and thus promotes derived evaluations. Taking both factors into account, the four conditions can be ranked according to the extent to which they encourage direct versus derived evaluations. The store-direct condition should produce the most direct evaluations; it both provides a meaningful characterization and a question focusing on stores. The store-derived condition should yield somewhat fewer direct evaluations; it provides a meaningful characterization, but it asks about items. The item-one-stage condition should yield few direct evaluations, because it does not include a meaningful characterization at all. Finally, the item-twostage condition explicitly requires that pattern of inter-item comparisons that should predominate in the item-one-stage condition.

A few final methodological details must be addressed. First, the order of the lone option and group was fully counterbalanced. Second, in each condition, every decision prob- 
lem appeared in four formats, varied between-subjects. Each format had a different one of the four items as the lone option. For example, in one format of the sandwich problem, turkey was the lone option, while ham, roast beef, and tuna salad formed the group; in a second format, ham was the lone option, while turkey, roast beef, and tuna salad formed the group, and so on.

For each format of each problem, we calculated the proportion of participants choosing the lone option. We refer to this proportion as the lone option choice share. The sum, $S$, of the four lone option choice shares across the four problem formats is a measure of group advantage or disadvantage. If consumers exhibit neither bias, $S$ should on average equal $100 \%$. Each lone option's share would correspond to its share in a choice among four ungrouped options, and those four shares necessarily sum to $100 \%$. Group advantage corresponds to $S<100 \%$ and group disadvantage to $S>100 \%$. Thus, hypothesis 1 predicts that $S$ will be less than $100 \%$ in the store-direct condition and greater than $100 \%$ in each of the item conditions:

H1a: $S_{\text {store-direct }}<100 \%$ (group advantage).

H1b: $S_{\text {item-one-stage }}, S_{\text {item-two-stage }}>100 \%$ (group disadvantage).

Moreover, we expect that $S$ will be smallest for the storedirect condition, somewhat larger for the store-derived condition, and larger still for the item-one-stage and item-twostage conditions:

$$
\text { H1c: } S_{\text {store-direct }}<S_{\text {store-derived }}<S_{\text {item-one-stage }} \leq S_{\text {item-two-stage. }}
$$

\section{Results and Discussion}

Table 1 displays the sum of lone choice shares $(S)$ for each problem and averaged across all eight problems. The results support each of the hypotheses. Across the eight problems, the average $S$ was $59 \%$ in the store-direct condition (significantly less than $100 \%, \mathrm{SE}=3.6 \%, z=$ $11.3, p<.001), 92 \%$ in the store-derived condition (marginally significantly less than $100 \%, \mathrm{SE}=4.4 \%, z=$ $1.9, p=.056), 116 \%$ in the item-one-stage condition (significantly greater than $100 \%, \mathrm{SE}=7.6 \%, z=2.1, p<$ .05 ), and $122 \%$ (significantly greater than $100 \%, \mathrm{SE}=$ $6.0 \%, z=3.7, p<.01)$ in the item-two-stage condition. Consistent with our predictions, every pair of conditions yields significantly different values of $S$ ( $p$ 's $<.01)$ except for the item-one-stage and item-two-stage conditions. Dependence of responses for each participant is accommodated by calculating, for each problem format, the standard errors of the average within-subject proportion of lone options selected. These standard errors are then aggregated across the four independent problem formats to get the overall standard error for each average value of $S$.

As can be seen in table 1 , the overall pattern for $S$ manifests itself in the individual choice problems as well. In all eight problems $S$ was greater in the item-one-stage than in the store-direct condition. All eight problems revealed an $S$ smaller than $100 \%$ in the store-direct condition, and six of the eight problems revealed an $S$ greater than $100 \%$ in the item-one-stage condition. Finally, six of the eight problems yielded the expected ordering of $S$ across the store-direct, store-derived, and item-one-stage conditions: lowest in store-direct, intermediate in store-derived, and highest in item-one-stage ( $p<.001$ by binomial test).

The observation of $S$ less than $100 \%$ in the store-direct and store-derived conditions replicates the pattern of group advantage found by Kahn et al. and Glazer et al. Furthermore, the results of experiment 1 sharpen these authors' conclusions, by contrasting marked group advantage $(S=$ $59 \%$ ) in the store-direct condition with minimal group advantage in the store-derived condition $(S=92 \%)$. Both conditions provide meaningful characterizations of $\mathrm{L}$ and $\mathrm{G}$ as stores, thus facilitating direct evaluation. Nevertheless, using an item-focused question to elicit preference in the store-derived condition encourages derived evaluations and, consistent with hypothesis $1 \mathrm{C}$, substantially diminishes the degree of group advantage.

The finding of $S$ greater than $100 \%$ in the item-one-stage condition replicates the finding of Brenner et al. Furthermore, the similar degree of group disadvantage for the itemone-stage $(S=116 \%)$ and item-two-stage conditions ( $S=122 \%$ ) supports these authors' assertion that the itemone-stage condition elicits the particular pattern of comparisons explicitly required by the item-two-stage condition. The similar results in the two conditions argue against the possibility that participants choose the lone option in the item-one-stage condition simply to conserve effort, or to reduce the difficulty of the choice problem by avoiding careful consideration of the options in the group.

In sum, the overall pattern of results reveals that the tendency to choose the lone option increases with the degree of derived evaluations and decreases with the degree of direct evaluations, thereby reconciling the opposite results of Kahn et al. (1987) and Brenner et al. (1999). Furthermore, the degree of direct and derived evaluations can be successfully influenced both by providing a meaningful characterization of choice options (as being located at different stores) and by adjusting the question used to elicit preferences.

\section{EXPERIMENT 2: PRICE SENSITIVITY}

Derived evaluations, by definition, look forward to alternatives available in subsequent choices, whereas direct evaluations do not. As a result, derived evaluations-but not direct evaluations - should be sensitive to variations in the characteristics of the individual items available in subsequent decisions. Experiment 1 was not able to test this fundamental prediction because the individual items available in subsequent decisions were held constant across all conditions.

In experiment 2, we again study choices among stores, but we now vary the prices of the individual items available 
TABLE 2

LONE OPTION CHOICE SHARES (\%) FOR EXPERIMENT 2

\begin{tabular}{|c|c|c|c|c|c|c|}
\hline Category & Lone option (prices) & Grouped options (prices) & $\begin{array}{l}\text { Derived } \\
\text { high price } \\
(n=38)\end{array}$ & $\begin{array}{l}\text { Derived } \\
\text { low price } \\
(n=35)\end{array}$ & $\begin{array}{l}\text { Direct high } \\
\text { price } \\
(n=38)\end{array}$ & $\begin{array}{l}\text { Direct low } \\
\text { price } \\
(n=34)\end{array}$ \\
\hline Cereal & Raisin Bran ( $\$ 4.29$ or $\$ 2.99$ ) & $\begin{array}{l}\text { Cheerios }(\$ 3.59) \\
\text { Frosted Flakes }(\$ 3.99) \\
\text { Grape Nuts }(\$ 3.29)\end{array}$ & 14 & 43 & 13 & 29 \\
\hline Entree & Chicken ( $\$ 4.09$ or $\$ 2.79$ ) & $\begin{array}{l}\text { Pasta }(\$ 2.99) \\
\text { Fish }(\$ 3.79) \\
\text { Beef }(\$ 3.39)\end{array}$ & 21 & 57 & 18 & 23 \\
\hline Beverage & Gatorade ( $\$ 1.49$ or $\$ .99)$ & $\begin{array}{l}\text { Fruitopia }(\$ 1.09) \\
\text { Snapple }(\$ 1.19) \\
\text { Frappuccino }(\$ 1.39)\end{array}$ & 39 & 71 & 45 & 35 \\
\hline Average $^{a}$ & & & $\begin{array}{c}25 \\
(4.3)\end{array}$ & $\begin{array}{c}57 \\
(5.2)\end{array}$ & $\begin{array}{c}25 \\
(4.3)\end{array}$ & $\begin{array}{l}29 \\
(5.2)\end{array}$ \\
\hline
\end{tabular}

a Standard errors are in parentheses.

at the stores. A change in the prices of individual items is not relevant to direct evaluations, such as "store A offers greater flexibility." On the other hand, a change in item prices should clearly affect derived evaluations of the stores. Consequently, participants should be more sensitive to changes in the prices of individual items when derived evaluations rather than direct evaluations predominate.

H2: In choices between stores, sensitivity to the prices of individual items should be greater under derived evaluation than under direct evaluation.

\section{Method}

Participants were 145 MBA students at a southeastern university who completed a short questionnaire as part of a classroom exercise. They made three choices, each from a different product category. As in the store conditions of experiment 1, participants were asked to imagine that they intended to purchase a single item from each category and to chose between a store offering one item and a store offering three items. Unlike experiment 1, participants were informed of the price of each item.

Two factors were manipulated between-subjects. First, based on the question used to elicit preferences, participants were encouraged to make either direct or derived evaluations of the stores. As in experiment 1, we compared store-direct and store-derived conditions; participants were asked either which store they preferred to visit or whether the item they most preferred was at the first or second store.

Second, an orthogonal manipulation varied the price of the lone item, making it either the least or most expensive of the four options. For example, in the low-price condition for the cereals category, one store offered Raisin Bran for $\$ 2.99$, while the other store offered Cheerios for $\$ 3.59$, Frosted Flakes for $\$ 3.99$, and Grape Nuts for \$3.29. In the high-price condition, the price of Raisin Bran was \$4.29, while the prices of the other three cereals remained the same. Hypothesis 2 predicts a particular interaction between the two manipulated factors: changing the price of the lone alternative should have a greater effect on its choice share in the store-derived than in the store-direct condition.

The specific prices and items used in each problem are presented in table 2. Because our aim was to detect changes in price sensitivity across direct and derived evaluations, rather than to demonstrate group advantage or disadvantage, there was no need to vary the lone item across four different problem formats. Thus, the identities of the lone item and the grouped items remained constant for all participants; only the price of the lone item and the focus on either direct or derived evaluations were manipulated. As a result, the data are presented as simple choice shares for the lone option rather than as sums of lone option choice shares $(S)$ across the four different possible lone options (as in experiment 1).

Although we did not vary the identity of the lone option, the use of a lone-versus-group partition is nevertheless important in the present experimental design. A lone-versus-group partition provides a rationale for direct evaluation of the group (such as preference for flexibility). Because participants are told so little about the stores, they have essentially no other basis for direct evaluation. Indeed, consider the consequences of a symmetric partition, in which each store carries two items. Symmetric partitioning would not differentiate between stores on the basis of flexibility and would thus provide no rationale for direct evaluation. In sum, by allowing direct evaluations based on a preference for flexibility, lone-versus-group partitioning allows for the store-direct versus store-derived manipulation to operate.

\section{Results}

The results, summarized in table 2, support the predictions of hypothesis 2 . In the store-derived condition the average lone option choice share was $25 \%$ when its price was high and $57 \%$ when its price was low, a difference of $32 \%$. In the store-direct condition, the average lone option choice shares were $25 \%$ (high price) and $29 \%$ (low price), a dif- 
ference of only 4\%. Price sensitivity was significantly greater in the store-derived condition than in the store-direct condition $(z=2.9, p<.01)$. Moreover, all three problems showed this same specific interaction. Participants were almost completely insensitive to the prices of individual items under direct evaluation but very sensitive to the prices of individual items under derived evaluation.

\section{GENERAL DISCUSSION}

Decisions that arise within a potential sequence of choices may involve direct evaluations (based on characteristics local to the current options) or derived evaluations (based on characteristics of individual options available in subsequent choices). Experiment 1 used the framework of direct and derived evaluations to reconcile opposite patterns of group advantage and disadvantage documented in the literature. Previously observed instances of group advantage (Kahn et al. 1987) may reflect direct evaluations favoring flexibility, whereas previously observed instances of group disadvantage (Brenner et al. 1999) may reflect derived evaluations. Experiment 2 examined how direct and derived evaluations influence price sensitivity. In store choices, sensitivity to changes in the prices of individual items was much greater under derived than under direct evaluations. Our experiments also indicate that the presence of a meaningful characterization and the question used to elicit preference are two ways to influence the reliance on direct versus derived evaluations.

\section{Factors Affecting the Impact of a Meaningful Characterization}

Experiment 1 varied the meaningful characterization of an option by the presence or absence of a store label, thereby encouraging either relatively more direct or derived evaluations. Many factors may influence the degree to which a meaningful characterization is recognized and affects the choice process. It is difficult to describe all the likely determinants of a meaningful characterization of a choice option, but we can note several attributes that such characterizations should share.

First, they should be unitary; the decision maker should be able to develop a single coherent picture of the choice option, rather than considering a collection of disparate images. This idea is analogous to a central aspect of basic level category representations (Rosch 1976). For example, the basic level category table is represented quite well by a single prototypical image, whereas the higher-order category furniture can not be so easily defined by a single prototypical image and naturally evokes a collection of images defined by the various salient subcategories (table, couch, dresser, etc.). Thus, table forms a single, coherent object of judgment, amenable to direct evaluation, whereas furniture prompts consideration of subcategories (which is analogous to derived evaluation). Similarly, a choice between purchasing either a table or a TV is likely to proceed more by direct evaluations than a choice between purchasing either a piece of furniture or a consumer electronics item.

Second, for a characterization of a choice option to be meaningful, it should be relevant or informative to the consumer's decision, in that the consumer can use the information that comes to mind in order to differentiate between the options. To illustrate, imagine a college senior who is considering whether to pursue a Ph.D. in psychology, pursue a Ph.D. in marketing, accept a job in management consulting, or accept a job in investment banking. Suppose that the student is asked to decide between getting a job and going to graduate school. If the student has few opinions about the overall merits of entry into the work force or continued schooling (i.e., the options get a job and go to graduate school), he or she may have little basis for direct evaluation. Thus, the student might instead engage in derived evaluations that focus on each specific job and each specific graduate program.

On the other hand, suppose that the student views pursuing a Ph.D. in marketing, accepting a job in management consulting, and accepting a job in investment banking as three ways of pursuing a business career, which stands in contrast to pursuing a career in psychology. In contrast to the earlier case (job vs. graduate school), the student may now have well-formed opinions about the overall merits of each alternative (e.g., my parents would frown on anything but a business career), and therefore direct evaluations may be quite salient. In these scenarios, the business/psychology representation provides characterizations to the decision maker that clearly differentiates the aggregate options, but the job/school representation does not. Thus, the tendency for direct evaluation is expected to be stronger in the business/psychology decision than in the job/school decision. Exploring the factors that allow some collections of choice options to have a coherent and informative unit identity is a promising area for future research.

\section{Heterogeneity in Direct and Derived Evaluations}

In our experiments, we have contrasted direct and derived evaluations with each other. We wish to note, however, that there will be substantial heterogeneity within both categories of evaluations and therefore each type of evaluation is likely to have diverse effects across different contexts. For instance, although direct evaluations yielded group advantage in experiment 1 , in other situations they may have the opposite effect, promoting group disadvantage. Rather than maintain maximum flexibility, people sometimes prefer to close off options when choices are very complex or seem overwhelming (Iyengar and Lepper 2000). When such preferences are operative, a greater reliance on direct evaluations would likely favor a smaller group of options over a larger group.

Derived evaluations may also have diverse manifestations and effects. Experiment 1, for instance, implicated a specific pattern of comparisons that starts within the group: options in the group were compared to one another, a favorite among the group options was identified, and the group favorite was 
then compared to the lone alternative. Because this procedure exposes the group favorite to many loss-emphasizing comparisons and the lone option to few such comparisons, it produces group disadvantage. But other patterns of comparisons are possible when derived evaluations occur, including some that may end up favoring the group. For example, consumers may sometimes focus on the lone option, comparing each member of the group to the lone alternative and selecting the lone alternative only if it prevails in all of such comparisons. Because this procedure exposes the lone option to many comparisons but each group member to just one comparison, it is expected to produce group advantage. Determining the patterns of comparisons consumers undertake in different choice contexts and examining the potential effects of these different forms of derived evaluation are worthwhile topics for further study.

The consequences of direct and derived evaluations will be very dependent on context; one cannot expect a consistent directional effect of direct evaluations across all circumstances. Nevertheless, we suggest that careful consideration of the different content of direct and derived evaluations will produce empirical predictions across many specific choice contexts (in addition to the two domains we have examined in experiments 1 and 2).

\section{Myopia and the Balance of Direct and Derived Evaluations}

In most situations, consumers will rely on some combination of direct and derived evaluations. We conclude by considering some evidence that consumers may typically rely too heavily on direct evaluations rather than derived evaluations. The results of the store-direct conditions in experiment 2 , in which people were essentially insensitive to price when direct evaluations were encouraged, indicate an apparent overemphasis on direct evaluations and an underemphasis on derived evaluations. In those experimental conditions, the prices of the products available at the stores ought to be an important consideration in store choice, but they were essentially ignored. Such overemphasis on direct evaluations and underemphasis on derived considerations can be considered a form of myopia, in which the characteristics of immediate options dominate the decision maker's attention and important but more distant considerations of subsequent options are neglected. There are numerous findings consistent with the view that decision makers are myopic in focusing too narrowly on immediate decisions and ignoring the broader context in which those decisions occur.

Kahneman and Lovallo (1993), for instance, found that decision makers tend to treat risky decisions in isolation, ignoring the fact that the future typically holds multiple opportunities for additional risky decisions. People often express different preferences when evaluating a single play of a gamble or multiple plays of the same gamble; a single opportunity to play a positive expected value gamble will frequently be turned down, even though the decision maker would gladly play the gamble multiple times. The narrow focus on a present decision (with a corresponding failure to consider future opportunities) therefore leads to excessive risk aversion. The myopic mistake here is that the decision maker does not look ahead to future decisions, which will likely offer the opportunity to embed or dilute the present decision within a more attractive portfolio of similar decisions (for a related result, see Benartzi and Thaler [1995]).

Camerer et al. (1997) found a similar myopic focus in the labor decisions of New York cab drivers. Instead of working more hours on good (i.e., passenger-heavy) days, and fewer hours on bad (i.e., passenger-scarce) days, cab drivers tended to work as long as was needed to achieve a daily income target. Thus, contrary to predictions of economic theory, the supply of cab drivers tended to be greater on bad days than on good days. This anomalous result appears to occur because the cab drivers focus too narrowly on achieving a daily income target rather than taking a broader view and attempting to achieve longer-term weekly or monthly income targets.

The myopic behaviors studied by both Kahneman and Lovallo (1993) and Camerer et al. (1997) arise when a single immediate choice is mistakenly isolated from an entire class of choices having the same essential features (playing multiple gambles, how much to work across multiple days). In both cases, more normatively appropriate behavior might result if the decision maker explicitly considered the entire class of choices rather than each individual choice in turn (e.g., by enacting a policy of always accepting small risks, by considering monthly wages rather than daily wages). The myopia uncovered in experiment 2 , however, is distinctly different from this isolated versus aggregated myopia of the preceding examples. The type of myopia shown in experiment 2 can be seen as somewhat more general in scope; it can occur whenever a single, immediate choice (e.g., between stores) is focused upon to the neglect of potential subsequent choices (e.g., between items). Note that, in general, the immediate choice and the potential later choices may involve fundamentally different types of choice options (e.g., stores vs. items) and consequently different considerations. In these types of cases, there is no clear way to subsume the sequence of potential choices into a coherent class of similar decisions, as would be possible in the Kahneman and Lovallo (1993) and Camerer et al. (1997) examples.

Among demonstrations of myopia, Hsee et al.'s (2003) study of myopic maximization perhaps most clearly indicates how people may rely too much on direct rather than derived evaluations and make suboptimal choices as a result. In one experiment by Hsee et al., participants chose between working on either a 6- or 7-min. task. In a control condition, the 6-min. task was rewarded with a pint of vanilla ice cream and the 7-min. task with a pint of pistachio ice cream. In a condition involving an intervening currency (or medium), the 6-min. task was rewarded with 60 points and the 7-min. task with 100 points. Participants could then exchange 60 points for a pint of vanilla ice cream or 100 points for a pint of pistachio ice cream. The 7-min. task was substantially 
more popular in the presence of the medium, despite the fact that the great majority of participants later indicated a preference for vanilla over pistachio ice cream. Hsee et al. explain these results by arguing that people attempt to maximize with respect to a salient medium (e.g., points) without sufficiently considering the outcomes they can subsequently obtain using that medium (e.g., ice cream).

Myopic maximization may be described in terms of direct and derived evaluations. As Hsee et al. (2002) point out, most decisions involve multiple layers of media. When one uses money earned from work to purchase a camera with which to take photos, the money, camera, and even the photos are all media separating the labor performed from the satisfaction or pleasure ultimately consumed. The decision about what work to perform engenders subsequent decisions about how to spend the money earned from work, which, in turn, yields subsequent decisions about how to use the camera on which that money has been spent. Each decision in the sequence may be made by the maximization of local considerations (i.e., direct evaluation) or by looking forward to and maximizing the outcomes of subsequent decisions (i.e., derived evaluation) or by some combination. One way to interpret myopic maximization, as well as other findings of myopic behavior, is that decisions frequently rely too heavily on direct evaluations compared to derived evaluations.

[David Glen Mick served as editor and Joel Huber served as associate editor for this article.]

\section{REFERENCES}

Benartzi, Shlomo and Richard H. Thaler (1995), "Myopic Loss Aversion and the Equity Premium Puzzle," Quarterly Journal of Economics, 110 (February), 73-92.

Bettman, James R. (1970), "Information Processing Models of Consumer Behavior," Journal of Marketing Research, 7 (August), 370-76.

- (1971), "The Structure of Consumer Choice Processes," Journal of Marketing Research, 8 (November), 465-71.

- (1979), An Information Processing Theory of Consumer Choice, Reading, MA: Addison-Wesley.

Bettman, James R. and C. Whan Park (1980), "Effects of Prior Knowledge and Experience and Phase of the Choice Process on Consumer Decision Processes," Journal of Consumer Research, 7 (December), 234-48.

Brenner, Lyle, Yuval Rottenstreich, and Sanjay Sood (1999), "Comparison, Grouping, and Preference," Psychological Science, 10 (May), 225-29.

Camerer, Colin, Linda Babcock, George Loewenstein, and Richard Thaler (1997), "Labor Supply of New York City Cabdrivers: One Day at a Time," Quarterly Journal of Economics, 112 (May), 407-42.

Glazer, Rashi, Barbara Kahn, and William Moore (1991), "The Influence of External Constraints on Brand Choice: The LoneAlternative Effect," Journal Of Consumer Research, 18 (June), 119-27.
Hauser, John R. (1986), “Agendas and Consumer Choice,” Journal of Marketing Research, 23 (August), 199-212.

Hsee, Chris and France Leclerc (1998), "Will Products Look More Attractive When Presented Separately or Together?" Journal of Consumer Research, 25 (September), 175-86.

Hsee, Chris, Frank Yu, Jiao Joe Zhang, and Yan Zhang (20023), "Medium Maximization," Journal of Consumer Research 30 (June), 1-14.

Huber, Joel, John W. Payne, and Christopher Puto (1982), “Adding Asymmetrically Dominated Alternatives: Violations of Regularity and the Similarity Hypothesis," Journal of Consumer Research, 9 (June), 90-98.

Iyengar, Sheena and Mark Lepper (2000), "When Choice Is Demotivating: Can One Desire Too Much of a Good Thing?" Journal of Personality and Social Psychology, 79 (December), 995-1006.

Kahn, Barbara, William Moore, and Rashi Glazer (1987), "Experiments in Constrained Choice," Journal Of Consumer Research, 14 (June), 96-113.

Kahneman, Daniel and Dan Lovallo (1993), "Timid Choices and Bold Forecasts: A Cognitive Perspective on Risk Taking," Management Science, 39 (1), 17-31.

Koopmans, Tjalling (1964), "On Flexibility of Future Preferences," in Human Judgments and Optimality, ed. M. Shelly and G. Bryan, New York: Wiley \& Sons, 243-56.

Kreps, David (1979), "A Representation Theorem for Preference for Flexibility," Econometrica 47 (May), 565-77.

Payne, John (1976), “Task Complexity and Contingent Processing in Decision Making: An Information Search and Protocol Analysis," Organizational Behavior and Human Performance, 16 (August), 366-87.

Peleg, Bezalel and Menahem Yaari (1973), "On the Existence of a Consistent Course of Action When Tastes Are Changing," Review of Economic Studies, 40 (July), 391-401.

Rosch, Eleanor (1976), "Basic Objects in Natural Categories," Cognitive Psychology, 8 (July), 382-439.

Simon, Herbert A. (1983), Reason in Human Affairs. Stanford, CA: Stanford University Press.

Slovic, Paul (1972), "From Shakespeare to Simon: Speculations and Some Evidence about Man's Ability to Process Information," Research Bulletin of the Oregon Research Institute, 12 (April), 1-29.

Slovic, Paul, Dale Griffin, and Amos Tversky (1990), "Compatibility Effects in Judgment and Choice," in Insights in Decision Making: A Tribute to Hillel J. Einhorn, ed. Robin Hogarth, Chicago: University of Chicago Press, 5-27.

Strotz, Richard (1955), "Myopia and Inconsistency in Dynamic Utility Maximization," Review of Economic Studies, 23 (3), 165-80.

Tversky, Amos and Daniel Kahneman (1986), "Rational Choice and the Framing of Decisions," Journal of Business, 59 (October), S251-S278.

(1991), "Loss Aversion in Riskless Choice: A Reference Dependent Model," Quarterly Journal of Economics, 106 (November), 1039-61.

Tversky, Amos, Shmuel Sattath, and Paul Slovic (1988), "Contingent Weighting in Judgment and Choice," Psychological Review, 95 (July), 371-84.

Tversky, Amos and Itamar Simonson (1993), "Context-Dependent Preferences," Management Science, 39 (October), 1179-89. 
Copyright of Journal of Consumer Research is the property of Journal of Consumer Research, Inc. and its content may not be copied or emailed to multiple sites or posted to a listserv without the copyright holder's express written permission. However, users may print, download, or email articles for individual use. 